\section{Toll-erating AD}

\section{By Lev Osherovich, Senior Writer}

Laval University and GlaxoSmithKline plc have shown that peripheral administration of the adjuvant monophosphoryl lipid A can help treat Alzheimer's disease in mice. ${ }^{1}$ Although the findings point to a potential immunomodulatory approach for addressing the neurodegenerative disorder, the pharma is now focusing on elucidating the mechanism before advancing the program.

The joint academic-industry team made the discovery while examining the interplay between the innate immune system and AD pathology. The group looked at how activation of toll-like receptor 4 (TLR4), a sensor of bacterial lipids, affects the activity of microglia, which are brain-specific innate immune cells.

The group found that monophosphoryl lipid A (MPL), a mild TLR4 agonist, can stimulate clearance of $\beta$-amyloid (A $\beta$ ) from the brains of mice and prevent the onset of AD symptoms.

"We have been working on priming microglia to remove $A \beta$ from the brain without triggering massive inflammation," said team co-leader Serge Rivest, professor of medicine at Laval.

"Our study shows for the first time that high doses of MPL, a mild immunopotentiator, induce beneficial activities in terms of amyloid clearance in mice," said the other co-leader, Daniel Larocque, in an e-mail to SciBX. Larocque is leader of Alzheimer's vaccine R\&D at the GlaxoSmithKline Vaccines unit of GSK.

The findings are the latest piece of evidence suggesting a key role for inflammatory signaling and innate immune cell activity in $\mathrm{AD}$ pathogenesis. Until recently, immunotherapeutic strategies for $\mathrm{AD}$ have focused on $\mathrm{mAbs}$ that directly hit $\mathrm{A} \beta$, but a spate of studies have suggested that stimulated microglia can clear up $A \beta .^{2-5}$

\section{Eat it up}

The team compared the effect of MPL and a much stronger adjuvant, lipopolysaccharide (LPS), in a microglia-derived cell line. MPL modestly promoted proinflammatory signaling, cytokine production and morphological changes, whereas LPS had a strong effect on all of these proinflammatory markers.

The mild inflammatory response caused by MPL proved beneficial, whereas the strong effect of LPS did not.

Among the microglial behaviors the team examined was the uptake of fluorescently labeled bacteria, which are targeted and ingested by activated microglia through a process known as phagocytosis. Treatment with MPL or LPS stimulated the uptake of fluorescent labeled bacteria into microglia, suggesting that both TLR4 agonists enhanced phagocytosis.

Activation of TLR4 in the periphery drew innate immune cells toward the edge of the brain. In vivo, intraperitoneal injection of MPL caused moderate levels of peripheral monocyte proliferation, activation and migration into the brain parenchyma and choroid plexus, which are brain structures associated with the blood brain barrier (BBB).

In contrast, LPS caused high levels of proliferation, activation and migration.

The surprise was that peripheral activation of TLR4 by MPL injection protected mice from $\mathrm{AD}$ pathology. The animals had decreased $A \beta$ accumulation in the hippocampus and increased cognitive function compared with vehicle-treated controls. LPS did not improve $\mathrm{AD}$ pathology.

Results were reported in the Proceedings of the National Academy of Sciences.

\section{Prophylactic potential}

Rivest thinks MPL could be used to slow AD progression in patients with early forms of the disease.

"Most clinical trials with vaccines or antibodies against $A \beta$ have not worked very well because they are intervening too late," he said. "Innate immune cells can recognize and clear $\mathrm{A} \beta$, so this could be used early in disease for prevention."

He noted that the mouse studies involved repeated high doses of MPL and that "we don't know if this dose would be tolerated in patients." He said dose-ranging studies are needed to find a minimal effective regimen in mice.

MPL, which is about 100 -fold less potent than LPS in inducing an inflammatory response, is a component of the adjuvant used for Cervarix, GSK's bivalent vaccine against HPV types 16 and 18. In the mouse AD studies, MPL was delivered at a 200-300-fold higher dose per body weight than Cervarix.

Larocque said GSK is testing the effect of MPL treatment, either alone or in combination with $\mathrm{mAbs}$ against $\mathrm{A} \beta$, in mouse models of more advanced AD.

It remains unclear how MPL activates innate immune cells and how this activation translates into clearance of $A \beta$. The biggest mystery is why peripheral treatment with MPL enhances brain clearance of $A \beta$ because neither MPL nor activated peripheral monocytes can enter the brain structures affected by AD.

"MPL does not diffuse in the brain because of the large size of the molecules. Moreover, we did not observe a significant increase in the number of peripheral monocytes going into the brain following repeated injections of MPL," said Larocque. "Therefore, there are no direct interactions of MPL and brain cells."

The idea that peripheral innate immune cells can affect AD is not without precedent. Larocque cited prior evidence that agonizing TLR9 also elicits protective effects in a mouse model of AD. ${ }^{6}$ 


\section{ANALYSIS}

\section{TARGETS \& MECHANISMS}

Larocque and Rivest suspect MPL causes brain microglia and peripheral monocytes to work together to clear up accumulated $A \beta$.

One possibility is that MPL works indirectly by triggering production of BBB-diffusible factors that communicate a proinflammatory signal from peripheral monocytes into the brain. Those signals presumably stimulate microglia to ingest $\mathrm{A} \beta$.

Another question is why both LPS and MPL promote proinflammatory microglial activity but only MPL ameliorates AD pathology.

Michael Heneka, professor of clinical neuroscience at the University of Bonn, said the in vitro phagocytosis studies suggest LPS is more effective than MPL at promoting uptake of A $\beta$ but MPL is more effective in the in vivo AD model.

“The compound they're using is 100 times less potent than LPS, but it's not that much less potent at inducing inflammatory cytokines in vivo," he said.

Heneka noted that the findings were consistent with emerging evidence that microglia can have different levels of proinflammatory activation that exert different effects on $\mathrm{AD}$ pathology. In December 2012, Heneka's team reported that blocking a variety of proinflammatory signaling pathways in microglia could ameliorate $\mathrm{AD}$ in mice. ${ }^{3,7}$

It is also unclear whether phagocytosis of $A \beta$ by microglia is the real reason for MPL's effect in AD. Heneka wanted to see pulse-chase studies showing that $\mathrm{A} \beta$ ingested by MPL-activated cells actually gets degraded rather than just building up inside of the cells.
GSK's sole AD compound in the clinic is rilapladib. The small molecule inhibitor of lipoprotein-associated phospholipase $A_{2}$ (PLA P $_{2}$; PAFAH; Lp-PLA 2 ) is in Phase II testing.

GSK has filed patents in connection to the discoveries described in the PNAS paper, and the licensing status of the IP is undisclosed.

Osherovich, L. SciBX 6 (4); doi:10.1038/scibx.2013.79

Published online Jan. 31, 2013

\section{REFERENCES}

1. Michaud, J.-P. et al. Proc. Natl. Acad. Sci. USA; published online Jan. 15, 2012; doi:10.1073/pnas.1215165110

Contact: Serge Rivest, Laval University, Quebec City, Quebec, Canada

e-mail: serge.rivest@crchul.ulaval.ca

Contact: Daniel Larocque, GlaxoSmithKline Vaccines, Laval, Quebec, Canada e-mail: daniel.a.larocque@gsk.com

2. Osherovich, L. SciBX 6(1); doi:10.1038/scibx.2013.3

3. Heneka, M.T. et al. Nature; published online Dec. 19, 2012; doi:10.1038/nature11729

4. Fulmer, T. SciBX 5(9); doi:10.1038/scibx.2012.221

5. Cramer, P.E. et al. Science 335, 1503-1506 (2012)

6. Scholtzova, H. et al. J. Neurosci. 29, 1846-1854 (2009)

7. Kummer, M.P. et al. J. Neurosci. 32, 17824-17829 (2012)

\section{COMPANIES AND INSTITUTIONS MENTIONED}

GlaxoSmithKline plc (LSE:GSK; NYSE:GSK), London, U.K. Laval University, Quebec City, Quebec, Canada University of Bonn, Bonn, Germany 